\title{
Un modèle de croissance en hauteur des arbres en pessière pure irrégulière de montagne
}

\author{
B Courbaud 1, F Houllier 2, C Rupe 3
}

1 CEMAGREF Grenoble, division protection contre les érosions, domaine universitaire, 2, rue de la Papeterie, BP 76, 38402 Saint-Martin-d'Hères Cedex;

2 ENGREF, laboratoire ENGREF/NRA de recherches en sciences forestières, unité dynamique des systèmes forestiers, 14, rue Girardet, 54042 Nancy Cedex;

3 ONF, service départemental des Hautes-Alpes, 5, rue des Silos, BP 96, 05007 Gap Cedex, France

(Reçu le 28 septembre 1992; accepté le 4 février 1993)

Résumé - Un modèle de croissance en hauteur des arbres en pessière pure irrégulière de montagne est présenté. De type "arbre dépendant de la distance", il permet de simuler pas à pas l'évolution d'une placette dans laquelle chaque arbre est considéré à la fois comme individu modélisé et comme compétiteur potentiel pour les autres arbres de la placette. Pour chaque pas de calcul de $t a ̀$ $t+\Delta t$, l'accroissement d'un arbre i est calculé par l'équation :

$$
\Delta \mathrm{H}_{i, t}=\mathrm{POT}_{i, t} \times \mathrm{RED}_{i, t} \text {, avec POT } \mathrm{PO}_{i, t}=\mathrm{f}\left(\mathrm{H}_{i, t}\right) \text { et } \mathrm{RED}_{i, t}=\mathrm{g}\left(\mathrm{OMBi}_{i, t}\right)
$$

$\Delta \mathrm{H}_{i, t}=$ accroissement en hauteur de l'arbre i de t à $\mathrm{t}+\Delta \mathrm{t}$; $\mathrm{POT}_{i, \mathrm{f}}=$ accroissement potentiel sans compétition; $\mathrm{RED}_{i, t}=$ réducteur de croissance = effet de la compétition; $\mathrm{H}_{i, t}$ hauteur de l'arbre $i$ à l'instant $t ; \mathrm{OMB}_{i, t}=$ indice d'ombrage reçu par l'arbre $i$ à $t$. Pour ajuster le modèle, l'histoire de la croissance d'une placette de 56 arbres située en forêt de Chamonix a été reconstituée. Le modèle de compétition a permis de représenter un tiers de la variabilité interindividuelle. Testé également de manière théorique, il s'est montré capable de reproduire la diversité des formes des courbes de croissance en hauteur rencontrée en forêt irrégulière et les évolutions de la structure d'une trouée forestière. Des perspectives d'utilisation de ce modèle sont présentées : simulation des modifications de la structure d'une placette et comparaison de différentes conditions de croissance.

modèle / épicéa / Picea abies / peuplement irrégulier / croissance en hauteur / montagne

Summary - A model for height growth of trees in uneven-aged spruce mountain forest. $A$ tree distance-dependent height growth model has been presented for multi-layered, monospecific (Picea exersa), mountain forests. It can simulate step by step the growth of every tree in a plot, each tree being considered as a model subject and as potential competitor for all the other trees in the plot. For each step of the computation, the increment of a tree is calculated by the equation:

$$
\begin{gathered}
\Delta H_{i, t}=P O T_{i, t} \times R E D_{i, t} \\
\text { with } P O T_{i, t}=f\left(H_{i, t}\right) \text { and } R E D_{i, t}=g\left(O M B_{i, t}\right)
\end{gathered}
$$

$\Delta H_{\mathrm{i}, \mathrm{t}}=$ height increment of tree number $\mathrm{i}$ from $\mathrm{t}$ to $\mathrm{t}+\Delta t ; P O T_{\mathrm{i}, \mathrm{t}}=$ potential increment without competition; $H_{\mathrm{i}, \mathrm{t}}=$ height of tree $i$ at time $\mathrm{t} ; R E D_{i, \mathrm{~F}}=$ reducer of growth $=$ effect of competition; $O M B_{\mathrm{i}, \mathrm{t}}=$ empirical index representing the shadow of the neighbouring trees.

The past growth of 56 trees in a plot selected in the forest of Chamonix (Haute-Savoie) has been reconstructed. When adjusted to these data, the model accounted for a third of the inter-individual variability in this plot. Theoretical testing of model enabled us to demonstrate that it is capable of gener- 
ating the diversity of the heigh-growth curves encountered in irregular forests, as well as modifications in the stucture of a forest gap. Potential uses of such a model have been presented: simulation of the changes in the structure of a plot and their effect on individual tree growth.

model / spruce / Picea abies / uneven-aged / heigh growth / mountain

\section{INTRODUCTION}

En forêt de montagne, l'étude des structures de peuplements et de leur évolution revêt une importance particulière. D'une part, ces structures présentent une grande diversité avec, notamment, tout un ensemble de "structures irrégulières" mal définies et dont la dynamique reste difficile à analyser et à prévoir. D'autre part, elles déterminent la capacité de la forêt à remplir ses multiples fonctions : production de bois, mais aussi rôles paysager et écologique ou fonction de protection des activités humaines. Une caractéristique essentielle que l'on peut favoriser et chercher à maintenir est alors la "stabilité" du peuplement définie à la fois comme la résistance instantanée aux perturbations (ex : chablis dus aux tempêtes) et la permanence à long terme de la forêt par renouvellement de la mosaïque forestière (Courbaud, 1991). Dans ces 2 aspects, la structure du peuplement est déterminante : elle conditionne la sensibilité aux agent météoriques et l'état de la forêt après perturbation (par exemple existence d'une régénération déja établie, de bouquets d'arbres encore présents...), elle conditionne également la répartition des facteurs tels que la lumière, l'espace et les ressources hydriques ou minérales au sein du peuplement, et donc sa croissance ultérieure.

Dans ce contexte, les questions posées par les gestionnaires concernent, d'une part, la connaissance de l'évolution naturelle des peuplements dans le cadre d'une gestion de plus en plus extensive et, d'autre part, la définition des zones d'actions prioritaires, ainsi que la nature même de ces actions.
Une première manière d'aborder ces problèmes consiste à concevoir une typologie des peuplements, basée sur la quantification du nombre d'individus par classe de dimension et de l'abondance de la régénération sur une surface limitée (de l'ordre de quelques dizaines d'ares), puis à approcher l'évolution des peuplements par une analyse synchronique en retraçant les transitions entre types de structures. Une telle démarche a, par exemple, été mise en œuvre en Franche-Comté (Bruciamacchie et al, 1991). Un travail de typologie des structures est également mené actuellement au CEMAGREF (centre du machinisme agricole, du génie rural, des eaux et des forêts) de Grenoble : il a pour but d'élaborer une clé de détermination principalement basée sur des critères qualitatifs (par exemple le nombre de strates) pemettant de caractériser la structure de placettes d'un quart d'hectare dont la mosaïque constitue le peuplement forestier (Rupe et Renaud, 1992).

Cette approche reste néanmoins insuffisante, pour 3 raisons : elle ne permet pas de restituer la répartition spatiale des arbres, qui constitue pourtant un élément essentiel de la structure des peuplements irréguliers dans la perspective de la caractérisation de leur stabilité ; elle ne fournit que des indications qualitatives sur les modes et vitesses d'évolution des structures; elle ne permet pas de comprendre la dynamique des peuplements en mettant en évidence les mécanismes qui déterminent l'évolution de la mosaïque dans l'espace et le temps.

II nous fallait donc élaborer un outil permettant de représenter la dynamique d'un peuplement éventuellement irrégulier avec 
une bonne description des interactions spatiales et temporelles, de comprendre et prévoir les évolutions de sa structure. La construction d'un modèle de croissance pour les forêts irrégulières nous est ainsi apparue nécessaire. Dans un premier temps, nous avons choisi de nous limiter à des cas assez simples:

- en nous focalisant sur les seuls phénomènes de croissance, c'est-à-dire en négligeant d'autres processus importants qui participent de la dynamique des peuplements, à savoir la mortalité ainsi que la fructification, la dissémination, la germination et l'installation des jeunes semis ;

- en concentrant nos efforts sur une classe restreinte de peuplements, en l'occurrence les pessières pures irrégulières.

Pour construire ce modèle de croissance, nous nous sommes inspirés de divers travaux portant sur des peuplements plus ou moins analogues aux futaies irrégulières de montagne : de modèles dendrométriques nord-américains conçus pour représenter la croissance individuelle dans des peuplements hétérogènes, inéquiennes et/ou mélangés (Leary, 1979) ; de modèles plus mécanistes visant à expliciter les phénomènes de concurrence en peuplements homogènes (Mitchell, 1975 ; Sloboda et Pfreundt, 1989 ; Ottorini, 1991) ou mélangés (Pretzsch, 1990) ; des observations et des interprétations qualitatives de Schütz (1969) sur la croissance du sapin et de l'épicéa dans les futaies jardinées.

\section{CARACTÉRISTIQUES DU MODĖLE}

\section{Choix du type de modèle}

Le choix du type de modèles, c'est-à-dire de l'entité modélisée (Houllier et al, 1991), $a$ été déduit des objectifs de l'étude.
L'analyse de la stabilité des peuplements requiert une bonne description de leur structure, c'est-à-dire de la distribution des caractéristiques individuelles des tiges (généralement représentée sous forme d'histogrammes) et de leur répartition spatiale.

Les questions posées ne concernent pas un peuplement particulier dont on connaîtrait l'état initial par un inventaire et dont on voudrait prédire l'évolution à 10 ou 20 ans. Elles portent plutôt sur la représentation des tendances évolutives de types de structures théoriques variés et sur l'évaluation des ordres de grandeur de leurs vitesses d'évolution. Il est en effet prévu que ce travail s'articule avec une typologie des peuplements basée sur une maille de $1 / 4$ d'hectare et valable pour l'ensemble des Alpes du Nord (Rupe et Renault, 1992).

Nous avons donc retenu le principe d'un modèle d'arbre dépendant des distances. La synthèse au niveau du peuplement (par exemple au niveau de la maille de1/4 d'ha) est possible puisque chaque individu est étudié par référence à ses voisins et grandit en peuplement. D'autre part, un tel modèle n'est pas contingent à des types de structures particulières pourvu qu'on prenne soin d'échantillonner des arbres placés dans des situations très variées de concurrence.

\section{Choix de la variable étudiée}

Dans un premier temps, nous avons choisi d'étudier la croissance en hauteur. Bien que délicate à modéliser et assez rarement étudiée (car moins faciles à mesurer que la croissance en diamètre), la croissance en hauteur individuelle est en effet un processus essentiel pour la caractérisation de la structure des peuplements irréguliers et de leur stabilité. De plus, elle est vraisemblablement moins dépendante du développement du houppier que la croissance en diamètre. Les connaissances ac- 
tuelles incitent en effet à construire des modèles basés sur le schéma de la figure 1, inspiré notamment des travaux de Mitchell (1975) et Pretzsch (1990) : la croissance en hauteur détermine la structure verticale, qui conditionne dans une large mesure la compétition interindividuelle et le développement des houppiers, qui eux-mêmes déterminent l'accroissement en grosseur de la tige.

\section{Représentation de la concurrence entre arbres}

L'utilisation d'indices statistiques de compétition basés sur les dimensions respectives des tiges a souvent donné des résultats mitigés (Tome et Burkhart, 1989). II a donc semblé intéressant de s'orienter vers des mécanismes plus proches d'une réalité écologique. Bien qu'il existe des modèles cherchant à intégrer de nombreux facteurs (Shugart, 1984), les modèles de compétition en forêt ont essentiellement porté pour l'instant sur le contact entre les couronnes (Mitchell, 1975) ou la compétition pour la lumière (Sloboda et Pfreundt,

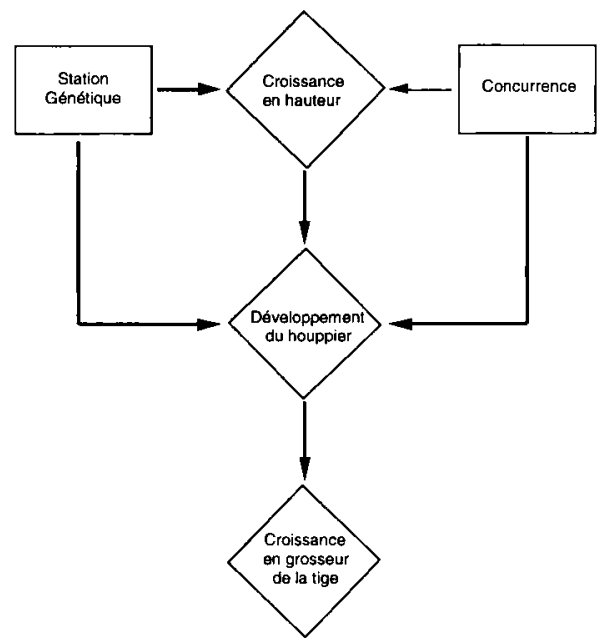

Fig 1. Principe des modèles de croissance de Mitchell (1975) et de Pretzsch (1990).
1989), parfois sur les 2 (Pretzsch, 1990). Dans un premier temps, nous avons privilégié le facteur lumière, généralement considéré comme de première importance et bien adapté à la forêt irrégulière dans laquelle la concurrence peut s'exercer à distance entre les arbres.

La croissance individuelle est par ailleurs influencée par des facteurs génétiques, ainsi que par les variations microstationnelles. Nous avons choisi de ne pas représenter ces 2 facteurs, parce qu'il est difficile d'en tenir compte dans les simulations autrement que par des fonctions aléatoires, parce que notre objectif était de mettre en évidence le rôle de la compétition dans l'évolution de la structure du peuplement, et parce que l'information nécessaire n'était pas disponible pour le peuplement étudié. Dans un premier temps, nous avons donc admis l'homogénéité génétique et stationnelle de la placette analysée (voir "Prise de mesures sur le terrain").

\section{MATÉRIEL ET MÉTHODE}

\section{Principe du modèle}

Le modèle repose sur une équation aux différences permettant de calculer les accroissements en hauteur pas à pas pour chaque période de croissance, tous les arbres étant traités en parallèle (fig 2).

Reprenant le principe de modèles d'accroissement en diamètre (Leary, 1979), nous avons représenté l'accroissement $\Delta \mathrm{H}_{i, t}$ de l'arbre $i$ de la date $t$ à $t+\Delta t$, par le produit d'un accroissement potentiel hors concurrence, $\mathrm{POT}_{i, t}$ et d'un réducteur tenant compte de l'effet de compétition, RED $_{i, t:}$

$$
\Delta \mathrm{H}_{i, t}=\mathrm{POT}_{i, t} \mathrm{RED}_{i, t}
$$

avec

$$
\mathrm{POT}_{i, t}=\mathrm{f}\left(\mathrm{H}_{i, t}\right) \text { et } \mathrm{RED}_{i, t}=\mathrm{g}\left(\mathrm{OMB}_{i, t}\right)
$$

où $\mathrm{H}_{i, t}$ : hauteur de l'arbre $i$ à $t$; $\mathrm{OMB}_{i, t}$ : indice d'ombrage reçu par l'arbre ià $t$. 


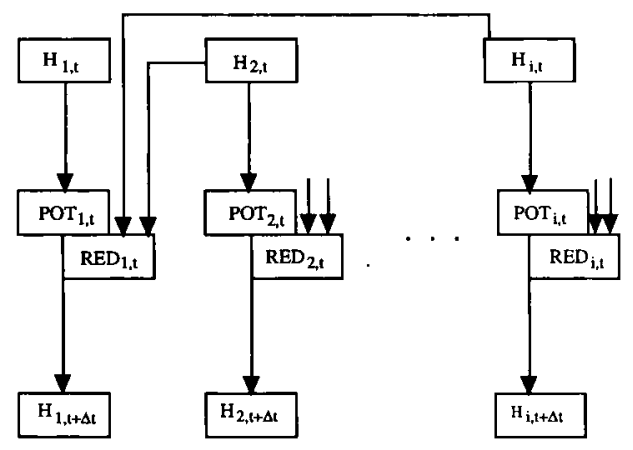

Fig 2. Principe du modèle. $H_{i t}$ : hauteur de l'arbre $i$ au temps $t, \mathrm{POT}_{i t}$ : accroissement potentiel de $t$ to $t+\Delta t ; R E D_{i t}$ : réducteur de croissance de $\mathrm{t}$ a $\mathrm{t}+\Delta \mathrm{t}$.

Le choix de travailler à partir d'une équation aux différences fonction de $\mathrm{H}$ présente 2 avantages :

- la durée de la phase de compression se traduit par un étalement très variable des courbes $\Delta H=f(t)$ qui est réduit à des différences minimes de tangente initiale par le passage aux courbes $\Delta H=f(H)$ (observation tirée des courbes de Schütz, 1969) ; ces dernières sont donc plus faciles à modéliser ;

- l'étude des accroissements permet d'autre part de travailler pas à pas en restant constamment en phase avec les variations de l'environnement.

L'histoire passée de l'arbre n'apparaît à aucun moment explicitement dans le calcul de son accroissement potentiel, seule sa hauteur intervient. Cette hypothèse biologique a été cependant acceptée dans un premier temps au regard de la faible variabilité des courbes $\Delta H=f$ (H).

Le réducteur est une fonction de l'indice d'ombrage total reçu par un arbre $i$ a la date $t$, $\mathrm{OMB}_{i,}$ qui résulte de la somme des ombrages apportés par chaque concurrent $j$, omb $\mathrm{om}_{i, t}$.

$$
\mathrm{OMB}_{i, t}=\sum_{j} \mathrm{Omb}_{i, j, t}
$$

Le modèle a été programmé en langage Pascal (programme Altitude, fig 3). Cette phase était indispensable pour ajuster les équations et tester le modèle.

\section{Prise de mesures sur le terrain}

\section{But des mesures}

La prise de mesures sur le terrain visait à reconstituer 1) la croissance en hauteur d'artores de hauteurs et de statuts sociaux variés en forêt irrégulière et 2) les conditions de concurrence ayant existé chaque année. L'accroissement en hauteur a été reconstruit dans le temps systématiquement pour tous les arbres d'une placette. Chaque arbre pouvait dont être considéré une année donnée comme sujet à modéliser et comme compétiteur pour tous les autres.

Un problème est posé par les arbres en bordure de placette, puisque nous ne disposons

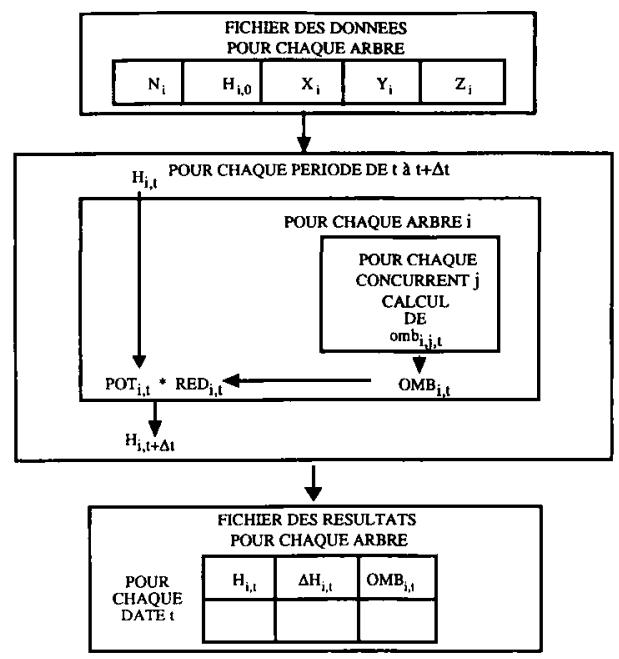

Fig 3. Structure du programme Atitude.

$N_{i}$ : numéro de l'arbre $i ; X_{i}$ : coordonnée de $i$ sur l'axe ouest-est; $Y_{i}$ : coordonnée de $i$ sur l'axe sud-nord; $Z_{\mathrm{i}}$ : coordonnée de $i$ sur l'axe vertical; $\mathrm{H}_{\mathrm{i}, \mathrm{t}}$ : Hauteur de $i$ à l'instant $t, \Delta \mathrm{H}_{\mathrm{i}, \mathrm{t}}$ : accroissement en hauteur de $t$ a $t+\Delta t, \mathrm{mb}_{\mathrm{i}, \mathrm{j}, \mathrm{t}}$ : ombrage de $j$ sur $i$ a l'instant $t, \mathrm{OMB}_{i, t}$ : ombrage total reçu par $i$ à l'instant $t, \mathrm{POT}_{\mathrm{i}, \mathrm{t}}$ : accroissement potentiel de $t$ à $t+\Delta t, R D_{i, t}:$ réducteur de croissance de $t$ à $t+\Delta t$. 
que d'une partie de leurs concurrents. Il a été partiellement résolu par 3 méhodes : nous avons préféré une placette unique de grande taille à plusieurs petites placettes, pour limiter les effets de bordure ; la placette choisie était limitée par des clairières naturelles sur deux de ses côtés ; enfin, les arbres de bordure ont été utilisés comme concurrents mais leurs accroissements ne sont pas intervenus dans l'ajustement statistique du modèle.

Pour être capable de reconstituer les conditions de concurrence ayant régné à chaque instant, nous nous sommes assurés que certains compétiteurs n'avaient pas disparu par mortalité au cours de la période mesurée.

\section{Choix du site}

Le site de mesure devait répondre aux critères suivants : situation dans les Alpes du Nord, pessière montagnarde pure, grande hétérogénéité dans les statuts sociaux des arbres et bonne homogénéité stationnelle. A posteriori, ce critère semble n'avoir été que partiellement satisfait (voir "Ajustement aux données de Chamonix»). Par ailleurs, il était impossible de s'assurer de l'homogénéité génétique du peuplement.

Le choix s'est porté sur le versant sud de la forêt de Chamonix. La placette retenue est situé à l'étage montagnard (altitude de $1165 \mathrm{~m}$ ) sur une forte pente $\left(38^{\circ}\right)$ et est exposée Sud/ Sud-Est (orientation: $150^{\circ}$ ). Elle se trouve dans une zone où la pessière pure correspond au climax (Rameau, communication personnelle). Le peuplement se présente comme une mosaïque extrêmement diversifiée où alternent des trouées de chablis, des zones de blocs rocheux et des taches de peuplement plus ou moins irrégulier. La placette présente une très forte hétérogénéité pied à pied de tailles, d'âges et de statuts sociaux des arbres. Ainsi, les arbres échantillonnés comportent aussi bien des arbres jeunes installés dans des tâches de régénération au sein d'une trouée que des arbres adultes isolés ou en petits bouquets. Au total, 56 arbres ont été mesurés : 46 sujets et 10 compétiteurs non sujets.

La forêt de Chamonix se caractérise actuellement par une gestion extensive orientée avant tout vers la récolte des chablis, la dernière exploitation ayant eu lieu sur la placette remonte à 35 ans, ce qui nous a autorisé à reconstituer son histoire sur les $\mathbf{3 0}$ dernières années.

\section{Technique de mesure}

Des analyses de tiges sur arbres abattus n'ayant pu être réalisées, chaque arbre de la placette a été grimpé pour mesurer les distances entre verticilles et reconstituer la croissance en hauteur. Les premiers verticilles à partir du haut étaient mesurés à l'aide d'une petite perche télescopique, un triple décamètre était ensuite accroché le long du tronc à l'aide d'un crochet souple et les verticilles suivants étaient mesurés en redescendant. L'observation fine de chaque verticille et l'analyse des cicatrices laissées par le bourgon terminal sur le tronc étaient ainsi possibles.

Au total, les verticilles groupés par 5 ont été mesurés sur 56 arbres de hauteur comprise entre 3 et $33 \mathrm{~m}$ pour les 30 dernières années.

La position de chaque arbre a ensuite été relevée selon 3 axes $X, Y, Z$.

\section{Ajustement}

\section{Méthode}

L'ajustement du modèle a été réalisé en utilisant à la fois une version adaptée du programme Altitude et le logiciel statistique SAS (SAS/STAT, 1987).

Le programme Altitude a permis de calculer differents indices empiriques d'ombrage pour chaque arbre sur les 30 dernières années, avec un pas de temps de 5 ans. Des équations ont ensuite été proposées pour la croissance potentielle et le réducteur. Elles ont été ajustées en bloc par régression non linéaire (procédure NLIN de SAS). Nous avons utilise 229 accroissements provenant des 46 arbres sujets.

\section{Modèle choisi}

Après avoir essayé plusieurs formulations pour les différents éléments du modèle (Courbaud, 1991), le meilleur ajustement a été obtenu pour le modèle suivant : les périodes d'accroissement sont de 5 ans.

L'accroissement potentiel est régi par une équation dérivée du modèle de ChapmanRichards (Pienaar et Turnbull, 1973).

$$
\mathrm{POT}_{i, t}=\frac{\mathrm{P} 2 \mathrm{P} 1^{(1-\mathrm{P} 3)}}{1-\mathrm{P} 3}\left(\mathrm{H}_{i, t}\right)^{\mathrm{P} 3}-\frac{\mathrm{P} 2}{1-\mathrm{P} 3} \mathrm{H}_{i, t} \text { (fig 4). }
$$


où $P 1, P 2, P 3$ : paramètres $(P 1$ représente la hauteur maximale, P2 est l'inverse d'une constante de temps et P3 conditionne la position du point d'inflexion de la courbe $H(t)$ ).

L'homogénéité génétique et stationnelle étant admise, la même courbe de potentiel en croissance libre a été attribuée à tous les arbres : P1, P2 et P3 sont supposés identiques.

La placette de mesure se situant sur un versant Sud marqué, nous avons considéré a priori comme compétiteurs tous les arbres situés au Sud d'une ligne Est-Ouest passant par le sujet $i$

$$
\begin{aligned}
& \Delta H(t): \text { Accroissement en hauteur } \\
& \text { sur } 5 \text { ans }(m)
\end{aligned}
$$

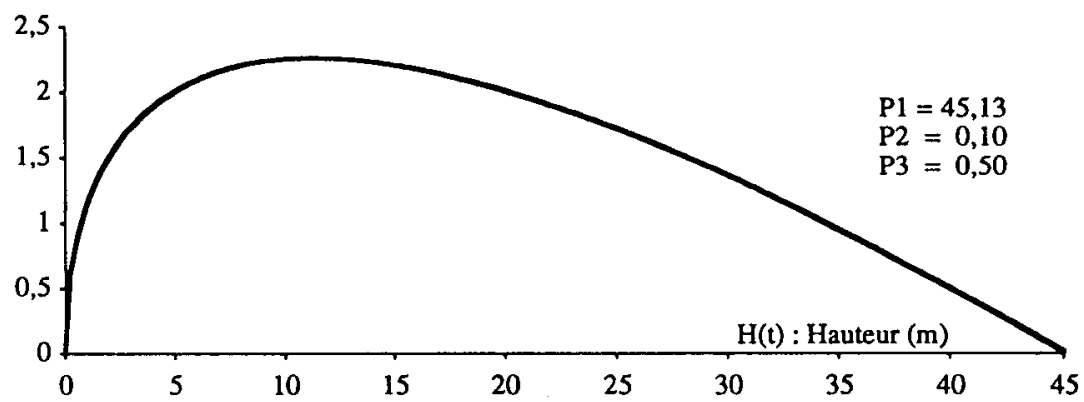

$\Delta H(t):$ Accroissement en hauteur
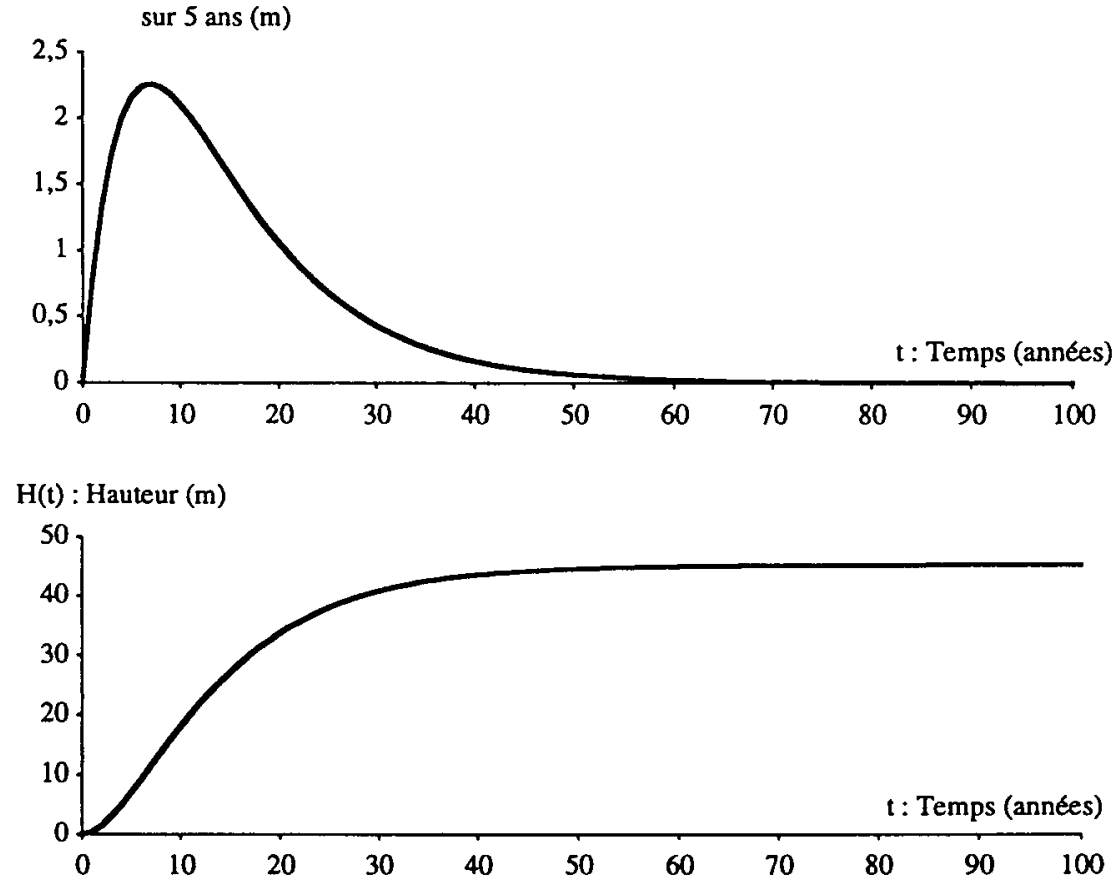

Fig 4. Croissance potentielle : différents aspects du modèle de Chapman-Richards. 
et visibles au-dessus d'un demi-plan horizontal passant à $80 \%$ de sa hauteur (fig 5). Le choix de cette hauteur a été fait sur des critères statistiques car c'est elle qui a permis d'obtenir le meilleur ajustement. Elle va dans le sens des connaissances sur la physiologie de l'arbre, qui indiquent que c'est l'ensemble des aiguilles de la partie supérieure du houppier qui produisent les photosynthétats utilisés pour la croissance en hauteur (Dickson, 1989).

La pente de la placette étant marquée $\left(38^{\circ}\right)$, les arbres situés au sud passent progressivement suivant leur taille en dessous du demi-plan horizontal de référence et ne sont donc plus considérés comme concurrents (en pratique les concurrents se trouvent tous à moins d'une vingtaine de mètres du sujet). Le rôle des arbres se trouvant dans des directions proches de l'est ou de l'ouest est, quant à lui, pondéré par l'azimut $\theta_{i, j}$ sous lequel ils sont vus jusqu'à s'annuler pour les directions plein-ouest et plein-est.

L'indice d'ombrage retenu est de la forme :

$$
\mathrm{omb}_{i, j, t}=\operatorname{tg} \varphi_{i, j, t} \cos \theta_{i, j}(\text { fig 5) }
$$

et

$$
\mathrm{OMB}_{i, j}=\sum_{j} \mathrm{Omb}_{i, j, t}
$$

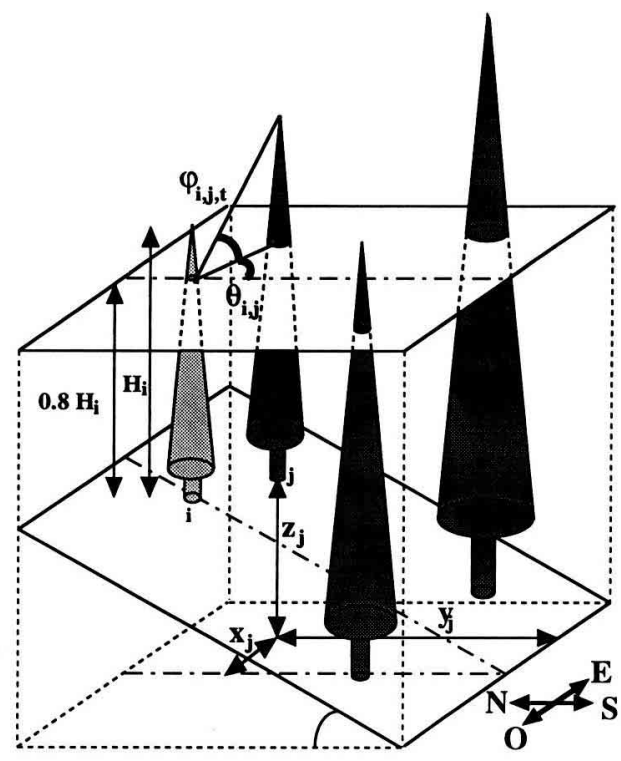

Fig 5. Calcul de l'indice d'ombrage où $\mathrm{OMB}_{i, t}$ : ombrage reçu par l'arbre $i$ à la date $t$; omb ${ }_{i, j, t}$ : ombrage apporté par le concurrent $j$ au sujet $i$ à la date $t ; \varphi_{i, j, t}=$ angle sous lequel le sommet de $j$ est vu depuis $80 \%$ de la hauteur de $i$ au temps $t ; \theta_{i, j, t}$ : azimut sous lequel $j$ est vu par $i-\pi / 2 \leq \theta_{i, t} \leq+\pi / 2$.

L'indice d'ombrage étant calculé à chaque instant à partir de la position et de la hauteur de tous les concurrents, il nécessite une récolte de données un peu délicate, cependant ces difficultés sont également rencontrées dès lors que l'on s'intéresse à la croissance en hauteur et participent de l'investissement inévitable pour aborder ce genre de problème.

Le réducteur, enfin, est de la forme:

$\mathrm{RED}_{i, t}=\exp \left(-\mathrm{R} 1 \mathrm{OMB}_{i, t}{ }^{2}\right) \quad \mathrm{R} 1>0 \quad(\mathrm{fig} 6)$

où $\operatorname{RED}_{i, t}$ : réducteur de croissance de l'arbre $i$ pour la période $t$ à $t+\Delta t ; \mathrm{R} 1$ : paramètre positif.

Pour des situations de compétition modéree il est généralement admis que la concurrence peut avoir un effet favorable sur la croissance en hauteur. Nous avons donc essayé une fonction qui soit maximale pour les ombrages faibles: $\mathrm{RED}_{i, t}=\mathrm{R} 0+(1-\mathrm{R} 0) \exp \left(-\mathrm{R} 1 \mathrm{OMB}_{i, t}{ }^{2}\right)$ avec $0 \leq R 0$. L'ajustement de cette fonction n'ayant pas produit de résultats sensiblement meilleurs (Ro n'était pas significativement différent de 0 au seuil de $5 \%$ ), nous avons préféré conserver le modèle le plus simple $(R 0=0)$.

\section{RÉSULTATS ET APPLICATIONS}

\section{Ajustement aux données de Chamonix}

Le meilleur ajustement est obtenu avec les paramètres présentés dans le tableau 1 .

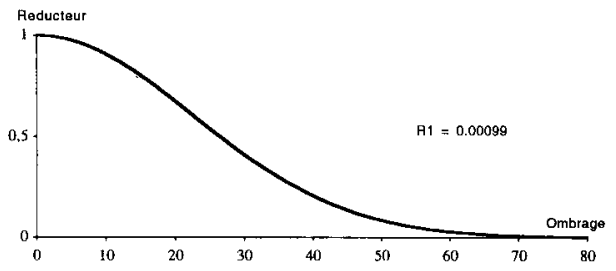

Fig 6. Réducteur de croissance RED ${ }_{i, t}$ 
Tableau I.

\begin{tabular}{ll}
\hline Estimation & $\begin{array}{l}\text { Intervalle de confiance } \\
\text { asymptotique a } 95 \%\end{array}$ \\
\hline$P 1=45,13$ & $40,28-49,98$ \\
$P 2=0,100$ & $0,077-0,124$ \\
$P 3=0,500$ & $0,495-0,504$ \\
$R 1=0,00099$ & $0,00057-0,00141$ \\
\hline
\end{tabular}

Fig 7a. Plan de la placette de Chamonix en 1991. Pente : $38^{\circ}$; orientation : $150^{\circ}$; altitude : $1165 \mathrm{~m}$. Hauteur comprise entre 0 et $10 \mathrm{~m}$; • hauteur comprise entre 10 et $20 \mathrm{~m}$; - hauteur comprise entre 20 et $30 \mathrm{~m}$. b. Hauteurs observées et calculées à partir de l'état initial 1956.

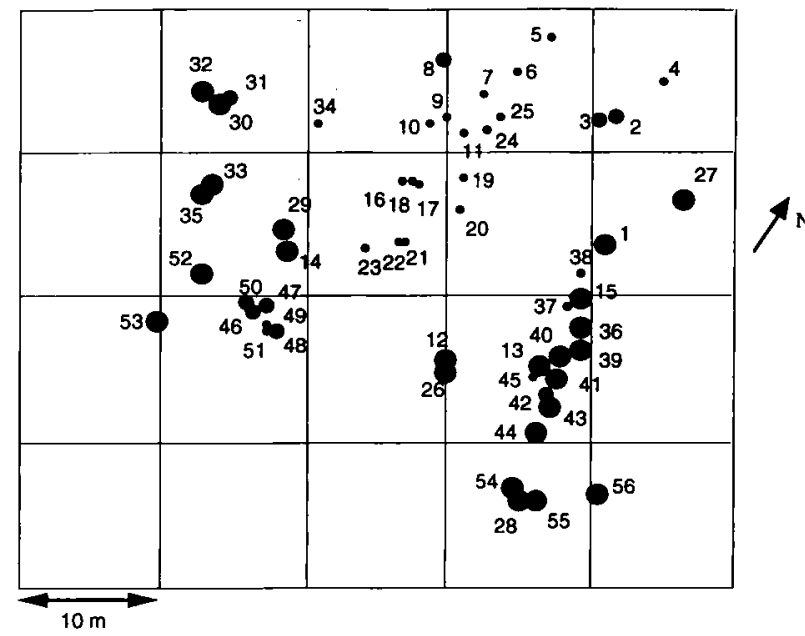

$\rightarrow-\mathrm{H}$ calculé

$\vec{\square}-\mathrm{H}$ réel
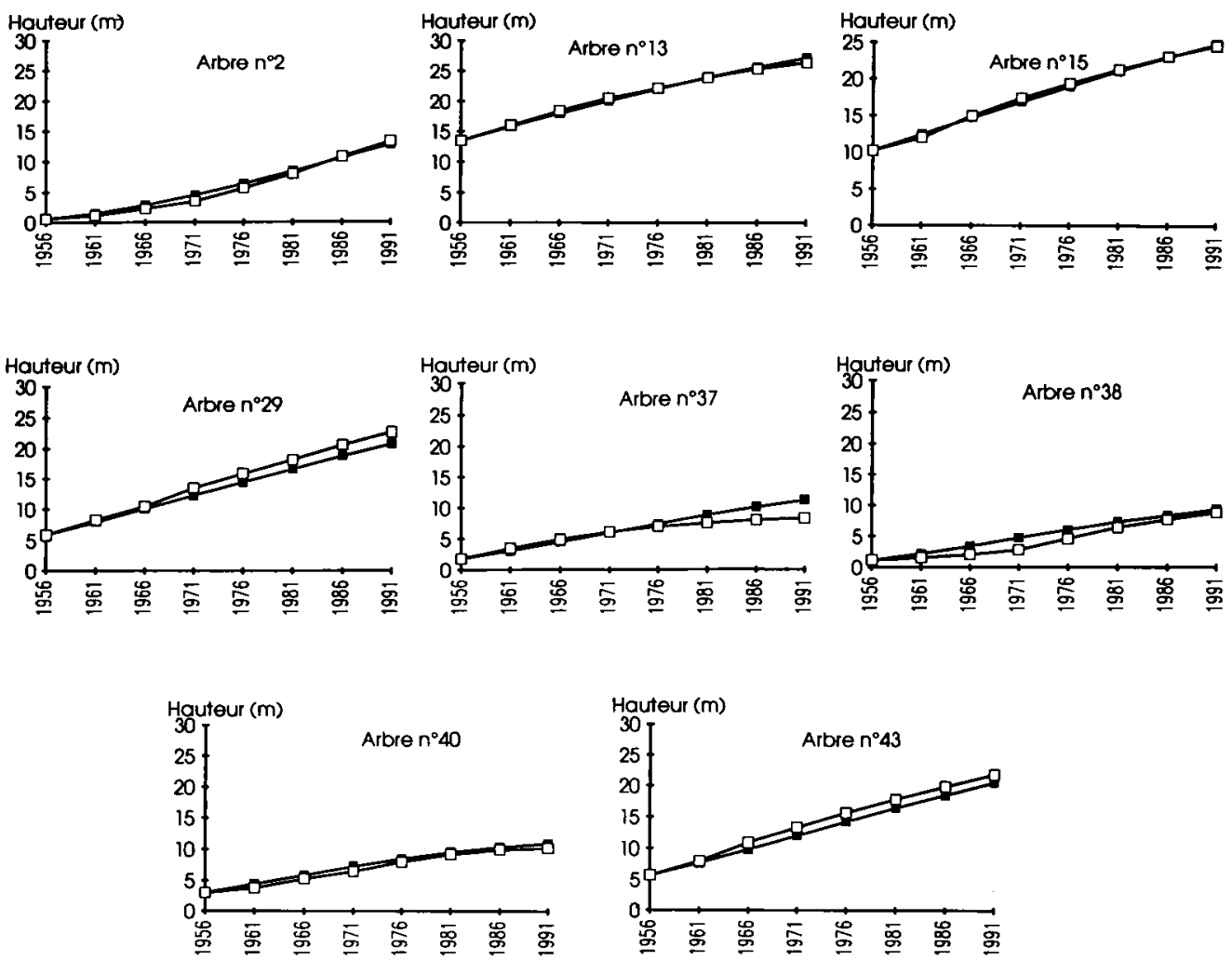
Avec 229 observations, on obtient un carré moyen des erreurs résiduelles de 0,39 , ce qui représente un écart type de $62 \mathrm{~cm}$ pour les accroissements sur 5 ans. Avec un modèle de croissance moyenne (modèle de Chapman-Richards, moyen seul sans réducteur) on obtenait un carré moyen des erreurs de 0,60 , soit un écart type de $77 \mathrm{~cm}$. L'introduction de l'indice d'ombrage et du réducteur de croissance a donc permis d'expliquer $1 / 3$ de la variabilité interindivididuelle $((0,60-0,39) / 0,60)$.

Notre approche simple de la concurrence pour la lumière au travers d'un indice pour l'instant encore largement empirique permet donc d'expliquer une part de la variabilité, mais reste insuffisante. La comparaison des croissances observées et des croissances calculées à partir d'un état initial en 1956 (fig 7) pour 35 arbres permet de constater que les courbes de croissance sont en général qualitativement bien représentées. Le biais moyen est de $0,7709 \mathrm{~m}$, avec une racine du carré résiduel moyen de $3,3480 \mathrm{~m}$. Le biais relatif moyen est de 0,1262 avec une racine de la moyenne du carré, résiduel relatif de 0,3884 .

On peut remarquer cependant que les arbres ayant des voisins de même statut qu'eux très proches (exemple $n^{\circ} 29, n^{\circ} 43$ ) ont tendance à être sous-estimés et que la croissance des arbres très dominés a tendance à être surestimée (exemples: $n^{\circ}$ $37, n^{\circ} 38, n^{\circ} 40$ ). L'introduction d'une compétition intrastrate pouvant avoir un effet positif sur la croissance en hauteur permettrait peut être d'affiner le modèle à ces différents niveaux.

Deux facteurs de variabilités importants ne sont par ailleurs pas pris en compte par le modèle : il s'agit des facteurs génétiques et surtout des conditions édaphiques du sol qui présentent une variabilité marquée en forêt de Chamonix avec un substrat d'éboulis.

\section{Simulations sur placette théorique}

\section{Structure de départ}

Le comportement du modèle a été analysé sur une placette théorique de structure plus simple que celle des placettes réelles. La structure initiale choisie symbolise une petite trouée de $12 \mathrm{~m} \times 12 \mathrm{~m}$ peuplée de petits arbres de $1 \mathrm{~m}$ de haut entourée par des arbres de $12 \mathrm{~m}$ de haut, espacés régulièrement de $3 \mathrm{~m}$ (fig 8 ). On s'intéresse à la croissance sur le transect médian A-B.
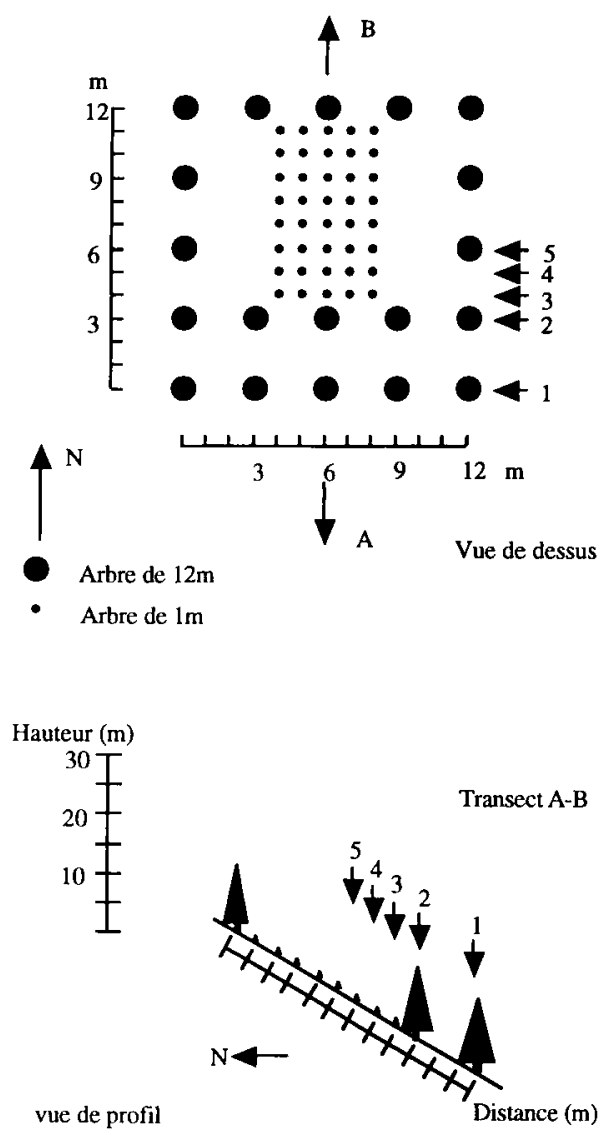

Fig 8. Simulation de la croissance dans une trouée : état initial. Pente : $30^{\circ}$ exposition sud, paramètre déterminés à Chamonix. 


\section{Variabilité des courbes de croissance (hors mortalité)}

La simulation fait apparaître 3 grands types de courbes d'accroissement en hauteur en fonction du temps, fréquemment rencontrés en forêt irrégulière (fig 9 ):

- les arbres subissant une concurrence faible présentent une courbe hauteur en fonction du temps classique en forme de cloche (courbe du potentiel) ;

- les arbres fortement dominés présentent une courbe en cloche de faible amplitude et atteignent rapidement sur leur hauteur maximale (arbre $n^{\circ} 3$ ou $n^{\circ} 4$ ) ;

- dans des cas intermédiaires, enfin, la courbe présente un replat sur sa pente descendante (arbre $n^{\circ} 5$ ). Ces formes originales, mises en évidence par les ana-
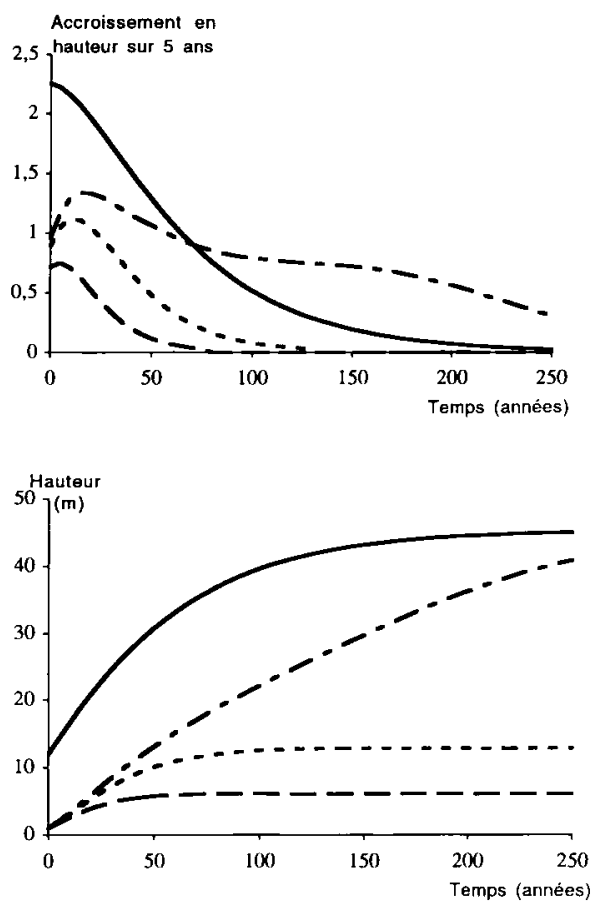

Fig 9. Transect A-B : diversité des courbes de croissance en hauteur. - arbre $n^{\circ} 2 ;-\ldots$ arbre $n^{\circ} 3$; - arbre $n^{\circ} 4$; - - - arbre $n^{\circ} 5$. lyses de tige de Schütz (1969) dans 2 futaies jardinées suisses et une forêt vierge yougoslave, ont été jusqu'à présent mal expliquées. Le modèle se montre capable de reproduire de telles courbes par le jeu de la concurrence. L'arbre $n^{\circ} 5$, concurrencé par les rangées $n^{\circ} 1$ et $n^{\circ} 2$, présente un accroissement maximal bien inférieur au potentiel. Les 2 premières rangées, approchant rapidement leur hauteur définitive, voient leurs accroissements diminuer fortement et même croiser la courbe de l'arbre $n^{\circ} 5$. Ce dernier, plus jeune, commence alors à présenter des accroissements supérieurs à ceux de ses concurrents et à se dégager progressivement de leur influence: il y a stabilisation de ses accroissements et replat sur la courbe.

\section{Modification de la structure le long d'un transect (hors mortalité)}

Les variations de la structure en hauteur du transect A-B au cours du temps peuvent également être analysées (fig 10).

Après une simulation de 25 ans, le transect présente une structure simple : plus les petits arbres de la trouée sont éloignés des lignes $n^{\circ} 1$ et $n^{\circ} 2$, plus leur croissance a été importante.

Dès le temps $t=30$ ans, les choses se compliquent car l'influence des voisins immédiats se superpose à celle de la lisière. L'arbre $n^{\circ} 5$ n'est pas gêné par le $\mathrm{n}^{\circ} 4$ car il a pris suffisamment d'avance sur lui dès les premiers pas de la simulation, le $n^{\circ} 6$ au contraire, à peine plus élevé que le $\mathrm{n}^{\circ} 5$ au temps $t=25$ ans, souffre de la concurrence de son voisin immédiat et se laisse progressivement distancer par lui. Par contagion, le transect des hauteurs prend alors une structure en dents de scie qui s'amortit avec la distance et avec le temps.

- Ces modifications de structures rappellent les observations de Schütz (1989) sur 

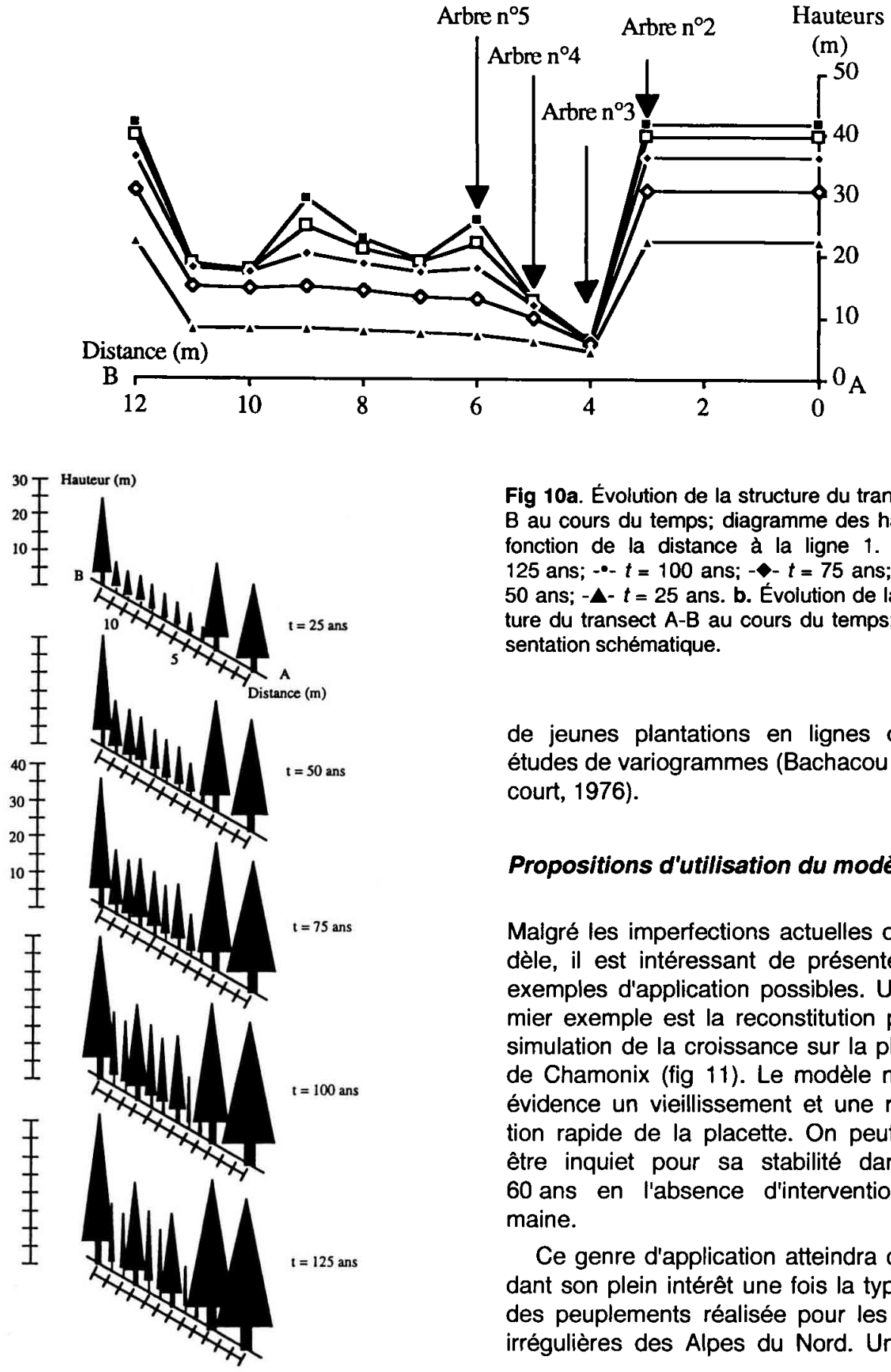

Fig 10a. Évolution de la structure du transect A$B$ au cours du temps; diagramme des hauteurs fonction de la distance à la ligne 1. - $-t=$ 125 ans; $-\bullet-t=100$ ans; $-t=75$ ans; $-t-t=$ 50 ans; $-\Delta-t=25$ ans. b. Évolution de la structure du transect A-B au cours du temps; représentation schématique.

de jeunes plantations en lignes ou les études de variogrammes (Bachacou et Decourt, 1976).

\section{Propositions d'utilisation du modèle}

Malgré les imperfections actuelles du modèle, il est intéressant de présenter des exemples d'application possibles. Un premier exemple est la reconstitution puis la simulation de la croissance sur la placette de Chamonix (fig 11). Le modèle met en évidence un vieillissement et une régulation rapide de la placette. On peut alors être inquiet pour sa stabilité dans les 60 ans en l'absence d'intervention humaine.

Ce genre d'application atteindra cependant son plein intérêt une fois la typologie des peuplements réalisée pour les forêts irrégulières des Alpes du Nord. Une fois 

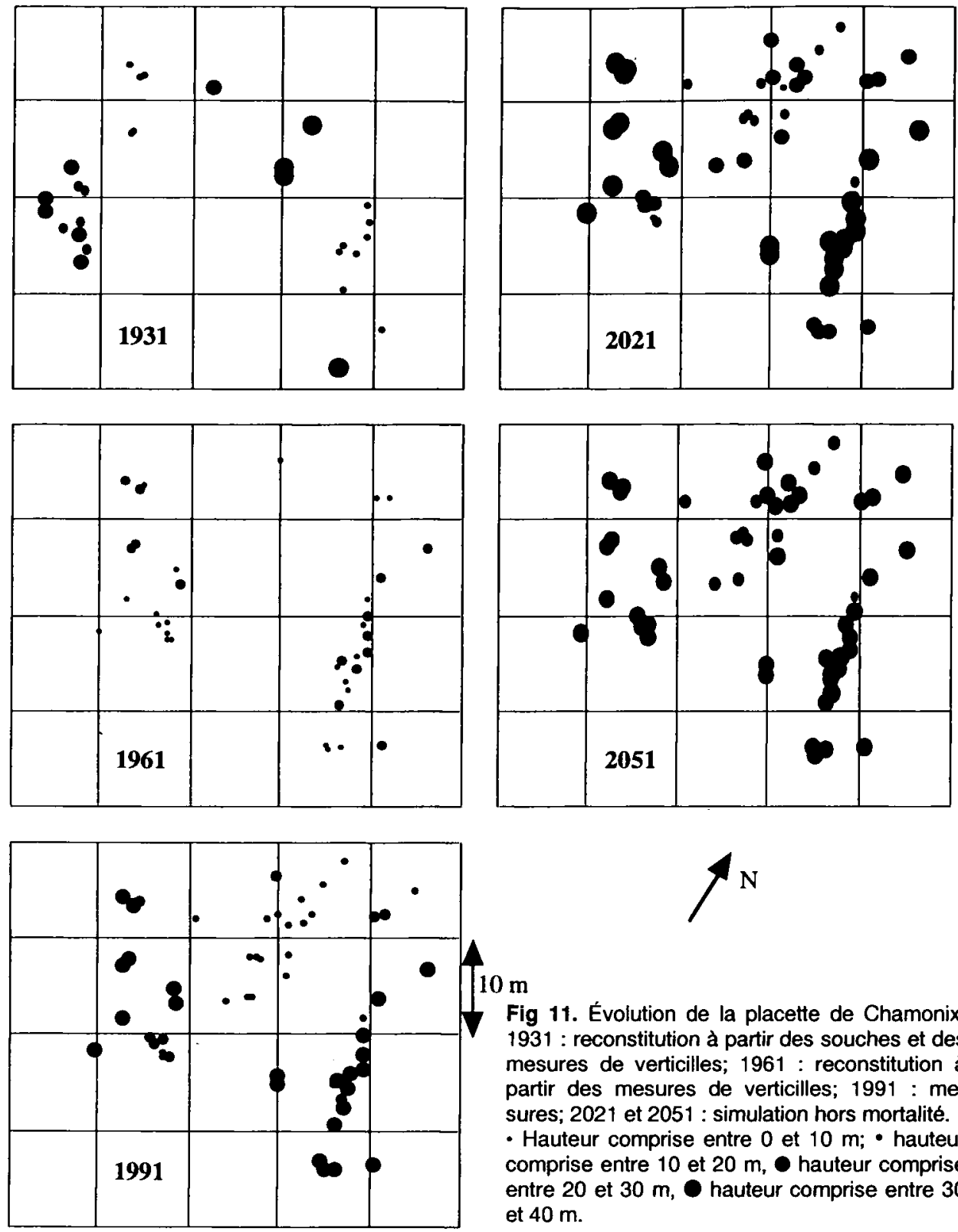

\section{$10 \mathrm{~m}$}

Fig 11. Évolution de la placette de Chamonix. 1931 : reconstitution à partir des souches et des mesures de verticilles; 1961 : reconstitution à partir des mesures de verticilles; 1991 : mesures; 2021 et 2051 : simulation hors mortalité.

- Hauteur comprise entre 0 et $10 \mathrm{~m}$; - hauteur comprise entre 10 et $20 \mathrm{~m}$, hauteur comprise entre 20 et $30 \mathrm{~m}$, hauteur comprise entre 30 et $40 \mathrm{~m}$. 
chaque type bien défini à l'échelle du $1 / 4$ d'hectare, il sera possible de proposer quelques structures théoriques stylisées représentant en quelque sorte l'archétype de chaque catégorie de peuplement. II sera alors aisé d'étudier ces structures modèles pour mettre en évidence les grandes tendances évolutives et avoir un bon ordre de grandeur de la vitesse à laquelle se transforment les peuplements. Les simulations permettront également de caractériser l'influence de petites variations de structure initiale sur leur dynamique.

On pourrait envisager par ailleurs d'étudier des influences telles que celles de l'exposition et de la pente sur la croissance, de 2 façons complémentaires : simulation sur différentes situations avec des paramètres donnés et ajustement du modèle dans différentes situations pour voir si les paramètres sont stables.

\section{DISCUSSION ET PERSPECTIVES}

\section{Améliorations possibles}

Différentes pistes se présentent pour améliorer le modèle. La représentation de la compétition doit être réalisée de manière beaucoup plus fine. II est indispensable pour cela de travailler sur la compétition pour la lumière mais également pour l'espace ou les ressources en eau. L'indice de compétition retenu permet ainsi de simuler une compétition à distance ou interstrate, mais la représentation d'effets plus fins comme l'action favorable d'un léger gainage sur la croissance en hauteur demanderait probablement l'élaboration d'un indice complémentaire, représentant la compétition de proximité ou intrastate. Le modèle doit par ailleurs être complété avec la représentation de la croissance du houppier et de la croissance en diamètre. La modélisation de la régénération et de la mortalité est enfin indispensable pour une simulation sur le moyen terme, car ces phénomènes influencent directement le nombre de compétiteurs et participent de la dynamique d'ensemble du peuplement.

Au niveau du matériel expérimental, des mesures plus fines, avec de vraies analyses de tige, sont envisagées, les placettes de mesure devant par ailleurs être multipliées avec des situations d'exposition et de structures variées, en cherchant à limiter la variabilité édaphique existant sur le site.

\section{Intérêt du modèle}

Malgré ses imperfections actuelles, ce modèle semble être une piste intéressante à poursuivre. Limité dans son principe à rendre compte de la variabilité interindividuelle de croissance en hauteur, due aux effets de la concurrence et du stade de développement (via POT $(\mathrm{H})$ ), son intérêt majeur réside dans des applications théoriques où les autres effets (génétiques, édaphiques...) sont éliminés.

II se montre ainsi un outil tout à fait intéressant, soit en relation avec la typologie des structures réalisée dans les Alpes du Nord, soit sur des structures fictives pour mieux analyser leur effet sur la croissance individuelle, ainsi que sur l'évolution-même de la structure.

\section{CONCLUSION}

L'élaboration d'un modèle de croissance relativement simple adapté à la forêt irrégulière s'avère possible, elle demande la mise au point d'un indice de compétition susceptible de représenter des concurrences à distance. Un indice complémentaire pour la compétition de proximité serait probablement d'apport bénéfique. $\mathrm{Ce}$ type de modèle peut permettre d'approfon- 
dir la compréhension de la dynamique des peuplements irréguliers, car il rend possible la comparaison des évolutions de structures théoriques de référence et, en jouant sur les paramètres, l'analyse de l'importance respective des différents éléments conditionnant la croissance.

\section{REMERCIEMENTS}

Nous tenons à remercier $L$ Confort et $D$ Rittie pour leur aide précieuse lors des mesures, ainsi que JC Rameau pour son analyse passionnante du contexte écologique de notre placette.

\section{REFÉRENCES}

Bachacou J, Decourt N (1976) Étude de la compétition dans des plantations régulières à l'aide de variogrammes. Ann Sci For 33 (4), 177-198

Bruciamacchie M, Groualle C, Minot P (1991) Modèle d'évolution des peuplements en futaie jardinée. Ann Sci For 48, 215-224

Courbaud B (1991) Introduction à une modélisation de la croissance en forêt irrégulière de montagne. DEA de biologie forestière, université Nancy / / ENGREF/ CEMAGREF

Dickson RE (1989) Carbon and nitrogen allocation in trees. Ann Sci For 46 suppl, 631s647s, Forest Tree Physiology, E Dreyer et al eds Elsevier/INRA

Houllier F, Bouchon J, Birot Y (1991) Modélisation de la dynamique des peuplements forestiers; état et perspectives. RFF 43, 87-107

Leary RA (1979) A general forest growth projection system applied to the Lake States region. USDA F-S, GTR NC-49, North Central Forest Experiment Station, 5-15
Mitchell KJ (1975) Dynamics and simultated yield of Douglas fir. For Sci Monogr 17

Ottorini JM (1991) Growth and development of individual Douglas-fir in stands for application to simulation in silviculture. Ann Sci For 48, 651-666

Pienaar LV, Turnbull KJ (1973) The ChapmanRichards generalization of Von Bertalanffy's growth model for basal area growth and yield in even-aged stands. For Sci 19(1), 2-22

Pretzsch H (1990) Strukturparameter zur steuerung von Wuchsmodellen für Rein-und Mischbestände. Deutscher Verband Forsdtlicher Forschungsanstalten. Sektion ertragskunde. Jahrestagung vom 28 , bis 30 , mai 1990. Verden/Aller

Rupe C, Renaud JP (1992) Stabilité et fonction de protection des forêts de montagne dans les Alpes du Nord - Approche méthodologique. Document CEMAGREF

SAS/STAT (1987) Guide for personal computers, version $6,1028 p$

Schütz JP (1969) Étude des phénomènes de la croissance en hauteur et en diamètre du sapin et de l'épicéa dans 2 peuplements jardinés et une forêt vierge. Thèse EPFZ Bühler buchdruck, Zürich

Schütz FP (1989) Zum Problem der Kondurenz in Mischbeständen. Schweiz Z Forstwes 140, $12,1069-1083$

Shugart $H$ (1984) $A$ theory of forest dynamics. Springer Verlag, Berlin

Sloboda B, Pfreundt J (1989) Tree and stand growth. A system analytical spacial model with consequences for test planing for thinning and single tree development. Artificial intelligence and growth models for forest management decisions. Publication $n^{\circ} \mathrm{FWS}-1-89$ School of forestry and wildlife resources, Virginia Polytechnic institute Blacksburg

Tome M, Burkhart HE (1989) Distancedependent competition measures for predicting growth of individual trees. For Sci 35, 3, 816-831 